\title{
Mindfulness training modifies subsystems of attention
}

\author{
Amishi P. Jha, Jason Krompinger, And Michael J. BAime \\ University of Pennsylvania, Philadelphia, Pennsylvania
}

\begin{abstract}
Mindfulness is defined as paying attention in the present moment. We investigate the hypothesis that mindfulness training may alter or enhance specific aspects of attention. We examined three functionally and neuroanatomically distinct but overlapping attentional subsystems: alerting, orienting, and conflict monitoring. Functioning of each subsystem was indexed by performance on the Attention Network Test (ANT; Fan, McCandliss, Sommer, Raz, \& Posner, 2002). Two types of mindfulness training (MT) programs were examined, and behavioral testing was conducted on participants before (Time 1) and after (Time 2) training. One training group consisted of individuals naive to mindfulness techniques who participated in an 8-week mindfulness-based stress reduction (MBSR) course that emphasized the development of concentrative meditation skills. The other training group consisted of individuals experienced in concentrative meditation techniques who participated in a 1-month intensive mindfulness retreat. Performance of these groups was compared with that of control participants who were meditation naive and received no MT. At Time 1, the participants in the retreat group demonstrated improved conflict monitoring performance relative to those in the MBSR and control groups. At Time 2, the participants in the MBSR course demonstrated significantly improved orienting in comparison with the control and retreat participants. In contrast, the participants in the retreat group demonstrated altered performance on the alerting component, with improvements in exogenous stimulus detection in comparison with the control and MBSR participants. The groups did not differ in conflict monitoring performance at Time 2 . These results suggest that mindfulness training may improve attention-related behavioral responses by enhancing functioning of specific subcomponents of attention. Whereas participation in the MBSR course improved the ability to endogenously orient attention, retreat participation appeared to allow for the development and emergence of receptive attentional skills, which improved exogenous alerting-related process.
\end{abstract}

Mindfulness has been defined as a process of "bringing one's complete attention to the present experience on a moment-to-moment basis" (Marlatt \& Kristeller, 1999, p. 68) and as "paying attention in a particular way, on purpose, in the present moment, and nonjudgmentally" (Kabat-Zinn, 1994, p. 4). Numerous meditation-based practices are used to train individuals to experience mindfulness. The most widely practiced form of mindfulness training (MT) is known as sitting meditation. In this technique, participants are instructed to sit in a relaxed, upright posture and to direct their full attention to the sensations of breathing. They are instructed to return their attention to the breath whenever it wanders. Thus, a fundamental aspect of MT is attentional training, and the task instructions of mindfulness techniques emphasize the role of attention.

Although MT has its roots in numerous cultural meditation practices (Wallace, 1999), it has recently become more widely available in medical contexts. Over 250 medical centers around the United States offer mindfulness-based stress reduction (MBSR) programs. These programs are typically conducted as courses that meet once a week for 8 weeks and teach participants to use mindfulness-based techniques (Kabat-Zinn, 2003; Kabat-Zinn et al., 1992). The effectiveness of MBSR for a variety of physical and psychological disorders has been examined in many studies (see Grossman, Niemann, Schmidt, \& Walach, 2004). There is growing evidence that MBSR is effective in the treatment of many conditions, including anxiety disorders, depression, fibromyalgia, chronic pain, substance abuse, binge eating, and skin diseases (Astin, 1997; Kabat-Zinn, 1990; Kabat-Zinn, Lipworth, \& Burney, 1985; KabatZinn et al., 1992; Kristeller \& Hallett, 1999; Marlatt \& Kristeller, 1999; Speca, Carlson, Goodey, \& Angen, 2000; Teasdale et al., 2000). In addition to MBSR programs, several other settings offer MT, including meditation centers and intensive retreat settings where mindfulness techniques are practiced for $10 \mathrm{~h}$ or more daily (Forte, Brown, \& Dysart, 1987-1988). Several studies report that MT in these contexts also results in health improvements (Brown, Forte, \& Dysart, 1984; Forte et al., 1987-1988; Page et al., 1997; Tori, 1999).

Thus, MT is finding increasing use as a tool to treat numerous psychological and physical maladies and to enhance health and quality of life (Grossman et al., 2004). Yet, surprisingly little is known about how MT affects the 
neural mechanisms that regulate cognition and emotion. Recent conceptualizations suggest that MT improves the self-regulation of attention (see Bishop et al., 2004). Although the systems that support attention are presumably involved, objective third-person measures of attention have seldom been used in research of MT. Instead, the bulk of studies have used introspection or standardized self-report data as dependent measures (see Grossman et al., 2004). There is much to gain from a more precise investigation of the role of attention in MT. If changes in attentional functions are associated with MT, further investigations could be conducted to explore whether or not these changes correspond to observable clinical benefits. This information could help clinicians to develop, implement, and evaluate mindfulness-based treatments. In addition, an understanding of the relationship between attention and MT could advance current cognitive neuroscience models of attention. That is, just as neuropsychological results enriched models of attention by providing findings that highlighted specific disease-related performance impairments, studies of MT may provide attentional findings that highlight training-related performance improvements. Such findings could lead to further exploration of cognitive-neural systems that are resilient to damage, amenable to reorganization, and capable of improving efficiency of processing through training or pharmacologic treatment.

Numerous writings suggest that MT improves two disparate forms of attention described as "concentrative" and "receptive" attention (Brown, 1977; Delmonte, 1987; Pfeiffer, 1966; Semple, 1999; Speeth, 1982; Valentine \& Sweet, 1999). In the former, attention is restricted to a specific focus, such as the breath. In the latter, attention is instead "objectless" and the goal is simply to keep attention fully "readied" in the present moment of experience without orienting, directing, or limiting it in any way. That is, attention is receptive to the whole field of awareness and remains in an open state so that it can be directed to currently experienced sensations, thoughts, emotions, and memories. Whereas extraneous stimuli are considered distractors in concentrative attention, in receptive attention no stimuli are extraneous because attention is open to the entire field of experience.

Many MT protocols recommend that concentrative attention should be mastered before receptive attention is cultivated (Kapleau, 1965). This recommendation is motivated by subjective reports from trainees that attention becomes lost in thought, mental images, or emotions (i.e., "mind wandering," as described by Smallwood \& Schooler, 2006) without the power of concentrative attention to keep it anchored to present experience (see Brown, 1977). Meditation texts posit that novices develop concentrative but not receptive attention skills early in the course of their training. More experienced meditators develop receptive attention as a consequence of continued concentrative training (see Lutz, Dunne, \& Davidson, in press, for a review). Importantly, some traditions further suggest that receptive attention cannot be explicitly trained but will emerge naturally after extensive experience with concentrative attention (Trungpa, 1975).
There are striking parallels between the concentrative/receptive dichotomy described in many meditation texts and recent cognitive neuroscience conceptualizations of dissociable dorsal and ventral attention systems. On the basis of behavioral, neuroimaging, lesion, and electrophysiological studies, Corbetta and Shulman (2002) proposed two partially segregated systems; a bilateral dorsal frontoparietal system involved in voluntary (top-down) orienting, and a right-lateralized ventral frontoparietal system involved in stimulus-driven (bottom-up) attention. The dorsal system is described as a voluntary attention system activated by presentation of cues indicating perceptual and response features of stimuli to which participants should direct their attention. In contrast, the ventral system is described as an alerting system activated during abrupt changes in sensory stimuli and detection of salient targets, especially when they are unexpected, are outside of the focus of attention, and have low probability of occurrence (Corbetta \& Shulman, 2002). Thus, dorsal system functions may be akin to concentrative attention, whereas ventral system functions may be analogous to receptive attention as described in meditation texts.

The dorsal/ventral model is similar to the tripartite model of attention proposed by Posner and Petersen (1990), according to which attention consists of three functionally distinct cognitive networks. These networks carry out the operations of alerting, orienting, and conflict monitoring. Alerting consists of achieving and maintaining a vigilant or alert state of preparedness, orienting directs and limits attention to a subset of possible inputs, and conflict monitoring prioritizes among competing tasks and responses. Recently, the attention network test (ANT) has been devised to identify behavioral and neural indices of alerting, orienting, and conflict monitoring during a single task (Fan, McCandliss, Sommer, Raz, \& Posner, 2002). Functional MRI studies of the ANT (see Fan, McCandliss, Fossella, Flombaum, \& Posner, 2005) report activation patterns during alerting that are highly overlapping with ventral attention system activity, as described by Corbetta and Shulman (2002). Both orienting and conflict monitoring activated dorsal system subregions corresponding to input-level selection and response-level selection, respectively. This division of the dorsal attention system into discrete subcomponents of input-level and response-level selection is consistent with information processing models of attention (Broadbent, 1970), which propose that both types of selection are forms of top-down voluntary attention. Input-level selection guides early sensoriperceptual analysis and corresponds to the orienting subsystem, whereas response-level selection guides decision and response processes during later information processing stages and corresponds to the conflict monitoring subsystem. This distinction could be helpful in clarifying meditation-related attentional changes, since concentrative meditation may influence input-level and/or response-level attentional selection. In the present study, we use components of the ANT to index attentional functions altered by MT. Specifically, we propose that concentrative attention skills can be indexed by behavioral markers of dorsal system functions (orienting and conflict 
monitoring) and receptive attention skills can be indexed by behavioral markers for ventral system functions (alerting and exogenous stimulus detection).

We conducted a behavioral study in three groups of participants. All the participants performed the ANT, and their response times (RTs) and accuracy scores were measured. One experimental group participated in an 8-week MBSR course that met once weekly for $3 \mathrm{~h}$. All members of this group were naive to meditation and received training in focusing attention on a single target, such as the breath, consistent with developing concentrative attention. The other experimental group participated in a fulltime 1-month mindfulness retreat. All members of this group had prior experience with concentrative meditation and received minimal group-level instructions on specific practices in addition to instructions to focus on the outbreath. The ANT was performed immediately before the start (Time 1) and shortly after completion of the MT program (Time 2) for both groups. Their performance was compared with that of control participants, who were also tested at two time points. We hypothesized that MT-related improvements on ANT performance would correspond to trials that index the aspects of attention that are cultivated and perhaps enhanced by MT.

Both traditional texts (see Lutz et al., in press, for a review) and previous reports (e.g., Valentine \& Sweet, 1999) suggest that concentrative attention is developed during the early stages of MT and that receptive attention develops in those more experienced with mindfulness practices. Thus, we hypothesized that MT may affect attentional subsystems differently in novices and in experienced mindfulness practitioners. To test this hypothesis, we investigated three main questions. First, do experienced mindfulness practitioners and novices differ in attentional functioning at Time 1? Since in the present study only the retreat participants had previous experience with concentrative meditation practices, our hypothesis was that their baseline performance would be better than that of the MT-naive participants on dorsal system functions such as orienting and conflict monitoring. Second, does participation in MBSR strengthen dorsal system functions? We hypothesized that since the MBSR group comprised novice meditators and instruction included development of concentrative skills, MT would allow this group to demonstrate improved dorsal system functioning in orienting and/or conflict monitoring relative to the control group at Time 2. Third, does participation in an intensive retreat strengthen either ventral or dorsal system functions? Since the retreat group already had considerable pretraining experience with concentrative meditation, one prediction was that intensive MT during the retreat would allow receptive attention to emerge. If so, the retreat participants might demonstrate improved ventral system functions, such as exogenous stimulus detection and alerting, relative to the participants in the control and MBSR groups at Time 2. Another possibility is that the retreat group could continue to improve in concentrative attention skills and demonstrate improved dorsal system functions at Time 2 relative to the control group and possibly the MBSR group.

\section{METHOD}

\section{Participants}

MBSR group. Seventeen participants (mean age $=24$ years, $S D=2.5$ ) were recruited from MBSR courses offered to medical and nursing students at the University of Pennsylvania. The participants were pooled across two course sessions with the same instructor, course content, and required meditation practice. This course was a variation of the public MBSR program offered by the Penn Program for Stress Management at the University of Pennsylvania School of Medicine. The course supplemented the standard course with additional content about communication and empathy in the health care setting. None of the participants had any prior experience with mindfulness techniques. Each 3-h class consisted of meditation practice, a group discussion, and interactive mindfulness-based exercises. The participants were also assigned $30 \mathrm{~min}$ of daily sitting meditation practice. The meditation practice initially emphasized attention to a single focus. For most concentrative exercises, this focus was the breath. The sensations of breathing were to be examined closely, and when attention wandered it was to be redirected back to the breath. In other exercises, the focus of attention was to be directed to sensations within specific body parts (body scan exercise) and sensations of walking (walking meditation). During the 5th week of classes, the MT was expanded to include some explicit training in receptive attention. The week-by-week content that the course followed has been described extensively for MBSR by Kabat-Zinn (1994).

Retreat group. The participants in the retreat group were taking part in an intensive 1-month residential mindfulness retreat held at Shambhala Mountain Center in Red Feather Lakes, Colorado. All the participants had previous concentrative meditation training (meditation experience prior to the retreat: range, 4-360 months; $M=60)$. The retreat included sitting and walking meditation and private interviews with experienced meditation instructors. Meditation instruction, although minimal, emphasized concentrative attention and directed the participants to place full attention on the out-breath. Much of the retreat was held in silence. The retreat participants engaged in formal mindfulness meditation practices for $10-12 \mathrm{~h}$ per day for the duration of the 30-day retreat. The age range of the participants was $22-57$ years $(M=35$ years, $S D=12.5)$. The content of group-level meditation instruction and individual instruction has been described extensively by Trungpa (1975).

Control group. The 17 participants in the control group were recruited from among medical and nursing students at the University of Pennsylvania and from the larger University community (mean age $=22$ years, $S D=2.3$ ). The control participants had no experience with meditation.

The study was approved by the University of Pennsylvania Institutional Review Board, and informed consent was obtained from each participant prior to entry into the study.

\section{Stimuli and Design}

Stimuli were presented via E-Prime (Version 1.2, Psychology Software Tools, Pittsburgh, PA) using a Dell Inspiron 4100 laptop. All the participants were given a variant of the ANT that was developed by Fan and colleagues and has been described in greater detail elsewhere (Fan, Fossella, Sommer, Wu, \& Posner, 2003; Fan et al., 2002; Fossella, Posner, Fan, Swanson, \& Pfaff, 2002). Briefly, the participants viewed a computer screen from a distance of $65 \mathrm{~cm}$, and their responses were collected via two input keys on a mouse. The participants were to respond using the index finger of each hand. A central fixation cross was part of the standing background throughout the experiment. With the exception of no-cue trials, all trials began with the presentation of a cue for $100 \mathrm{msec}$. The cue offset was followed by a brief (400-msec) delay interval. Each trial ended with the presentation of a target that appeared $1.068^{\circ}$ above or below fixation. The target remained on the screen until a response was executed but for no longer than $1,700 \mathrm{msec}$. The intertrial interval (ITI) varied randomly from 400 to $1,600 \mathrm{msec}$ across trials. On no-cue trials, $100 \mathrm{msec}$ with no stimuli were added to the ITI so that the total trial durations for all trial types were comparable. 
Target stimuli consisted of a row of five arrows (see Target Conditions in Figure 1). The participant's task was to determine whether the center arrow pointed in the leftward or rightward direction while maintaining fixation on the central cross throughout the trial. The participants responded with the index finger of each hand and were to press the left or right button on a mouse to indicate each response. The center arrow was flanked by four arrows (two to the right and two to the left) that pointed either in the same direction as the center arrow (congruent target) or in the opposite direction (incongruent target). Each target presentation was preceded by one of four cue conditions (see Cue Conditions in Figure 1): (1) During no-cue trials, the fixation point remained on the screen during the cue presentation period and no cue was presented. (2) During double-cue trials, asterisks simultaneously appeared at target positions above and below fixation. (3) During center-cue trials, a single asterisk appeared at fixation. (4) During spatial-cue trials, a single asterisk appeared at the location of the upcoming target. The spatial cue was $100 \%$ predictive of the target position and was equally likely to occur above or below the fixation point. These cue conditions varied in the temporal and spatial information they provided about the subsequent target presentation. No-cue trials provided neither temporal nor spatial warning; the target simply appeared. Double- and centercue trials provided only temporal warning, and spatial-cue trials provided both temporal and spatial information about the upcoming target. Thus, the experimental design comprised two within-subjects factors: cue type (no cue vs. double cue vs. center cue vs. spatial cue) and target type (congruent vs. incongruent). Each participant completed a 24-trial practice session prior to performing the experiment. The participants performed 288 experimental trials (72 per cue condition) during each testing session, which lasted $25 \mathrm{~min}$, including practice trials and short breaks. All trial types were randomly presented.

The participants in all three groups (MBSR, retreat, and control) participated in two testing sessions (Time $1 \mathrm{vs.} \mathrm{Time} \mathrm{2).} \mathrm{The} \mathrm{retreat}$ participants were tested at the beginning of the 1-month retreat and

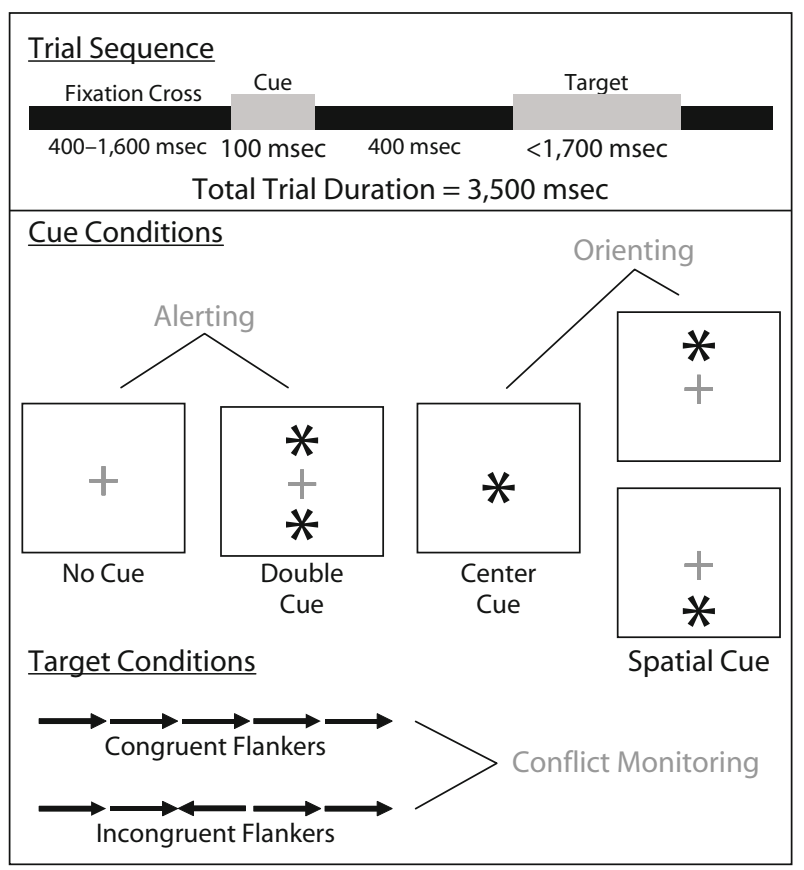

Figure 1. Trial sequence and timing for the attention network test. Participants were instructed to keep their eyes fixated on the center cross during all trials. The experiment consisted of four cue conditions and two target conditions. All trial types were randomly intermixed and equally probable. again at the end of the retreat (30 days after Time 1 testing). The MBSR and control participants were tested at two time points as well. The first testing session occurred before the beginning of the MBSR class, and the second session was completed within 10 days of the conclusion of the class (mean number of days between testing sessions, 59; $S D=8$ ).

\section{Data Analysis}

All analyses were performed on RT scores for correct trials only and accuracy (percent correct) scores. Performance for each attentional subsystem (alerting, orienting, and conflict monitoring) was examined separately by paired subtractions across subsets of conditions. Alerting was indexed by subtracting performance measures on double-cue trials from those on no-cue trials. Orienting was indexed by subtracting performance measures on spatial-cue trials from those on center-cue trials. Conflict monitoring was indexed by subtracting performance measures on congruent target trials from those on incongruent target trials. The results of these paired subtractions will be referred to as subsystem difference scores. This method of analysis has been extensively used with the ANT and has been reported in detail by Fan et al. (2002). Subtractions were performed for each participant at both time points for all three subsystems.

Analyses were performed to investigate three main questions. First, are there differences in performance between the participants who were experienced in (retreat group) and those who were naive to (MBSR and control groups) concentrative meditation before MT? Second, after MT, are there changes in dorsal system functions of orienting and/or conflict monitoring in either the MBSR or the retreat participants, who received instructions in concentrative meditation, relative to the control participants, who received no MT? Third, after MT, are there differences in ventral system functions of exogenous stimulus detection and alerting between the meditationexperienced retreat group and the groups with less (MBSR group) or no (control group) training? To answer these questions, we performed a series of planned contrasts and ANOVAs. Importantly, contrasts were conducted to conserve orthogonality and appropriate degrees of freedom and were corrected for multiple comparisons when appropriate. In addition, tests of homogeneity of variance were conducted to determine the magnitude of between-subjects variability in each group. The specific analyses described below were performed on RT and accuracy difference scores unless explicitly stated otherwise.

Time 1: The influence of prior experience with meditation. We test the hypothesis that prior experience with concentrative meditation may strengthen attention. Only the participants in the retreat group had prior experience with meditation. Since neither the MBSR nor the control group had any prior experience with meditation, these groups were not hypothesized to differ from each other in performance at Time 1. An initial contrast was made between the performance of the MBSR group and that of the control participants. In the cases in which these groups did not significantly differ from each other, they were jointly compared with the retreat group. This series of contrasts was conducted for difference scores on alerting, orienting, and conflict monitoring.

Time 2: The influence of mindfulness training. We investigated whether or not MT had an influence on the functioning of the dorsal and ventral systems in our participants and discuss each of these two cases in turn.

Does MT alter dorsal system functioning? We tested the hypothesis that MT with an emphasis on concentrative attention may improve dorsal system functions of orienting and/or conflict monitoring. For the MBSR group, we predicted that the introduction and cultivation of concentrative attention in the MBSR course might lead to MT-related improvements in dorsal system functions. For the retreat group, we hypothesized that instruction in concentrative attention during the retreat might also lead to improvements in dorsal system functions. The critical comparisons of interest were between each of these MT groups and the control group. Since the training contexts and the pre-MT meditation experience of the participants 
differed across groups, a comparison between the MBSR and retreat groups was of interest as well.

To determine whether or not performance differed across these groups, we performed two separate ANOVAs, each with a single factor of group (MBSR vs. retreat vs. control). One ANOVA was conducted to investigate the impact of group on orienting difference scores, and the other to investigate the impact of group on conflict monitoring scores. In addition to these ANOVAs, a series of paired contrasts was made to determine whether or not any pairs of groups differed from each other. Significance was determined after correction for multiple comparisons.

Does MT alter ventral system functioning? We tested the hypothesis that MT in experienced meditators could lead to cultivation of receptive attention, which may alter ventral system functions of alerting. Since only the retreat group had prior meditation experience, we predicted that MT would strengthen cultivation of receptive attention more significantly in this group than in the other groups. Emergence of receptive attention was not predicted for the MBSR or control participants, since they were novices to meditation or meditation naive. A series of paired contrasts was performed. In an initial contrast, the alerting difference scores for the MBSR group were compared with those of the control group. If no significant differences were found between these groups, their combined performance was compared with the performance of the retreat group. We predicted that the retreat participants might be better able than the MBSR and control participants to detect targets when no cues are provided, because the retreat training may have altered their ventral attention system to be in a more readied and receptive state for detection of exogenous events.

Time 1 versus Time 2: Influence of task repetition on performance. An ANOVA was conducted with two factors - Time (1 vs. 2) and group (retreat vs. MBSR vs. control) - for raw RTs and accuracy scores collapsed across all attention subcomponents, to determine if there were overall performance changes over time.

Influence of age. Previous attention studies have reported a decline in attentional efficiency and overall speed of response with age (Reuter-Lorenz \& Stanczak, 2000). It is important to note that the retreat group, unlike the MBSR and control groups, had a much broader participant age range (22-57 years, $M=35$ ). Therefore, age, in addition to training-related effects, was considered a possible factor influencing the pattern of results observed. The participants were grouped into older and younger subgroups on the basis of a median split analysis of participant age. In a paired contrast, overall RT and accuracy scores (collapsed across conditions) and component difference scores were compared for older and younger participants to test the hypothesis that RTs are longer in older participants than in younger participants. After this median split, the ages of the younger retreat group participants were compared with the ages of the control and MBSR participants to determine whether or not age differed significantly across groups.

Influence of meditation experience. The retreat group had a broad range of experience with meditation prior to the retreat (4-360 months, $M=60$ ). Since the prediction that ventral system functions are altered after retreat participation hinges on the retreat group's having prior experience with meditation, it is possible that the amount of prior experience may greatly influence performance observed at Time 2. To investigate this issue, we performed a correlation analysis for the retreat group in which months of meditation experience was correlated with task performance.

\section{RESULTS}

\section{Time 1: The Influence of Prior Experience With Meditation}

RT and accuracy difference scores were comparable to mean values previously reported by Fan et al. (2002). Overall task accuracy was very high $(97 \%, S D=2 \%)$. All analyses were performed on difference scores for both
RT and accuracy for each pair of conditions comprising each attention subsystem. Levene's test for homogeneity of variance was not significant for any of the subsystem difference scores for either RT or accuracy across groups $(p>.2)$.

Alerting. The first contrast between the MBSR group and the control group revealed no significant difference across groups in either RT difference $[t(1,32)=1.22$, $p>.2]$ or accuracy difference $[t(1,32)=0.92, p>$.3] scores. In the second contrast, in which these groups were collapsed, their performance was compared with that of the retreat group. No significant differences across these groups were present for RT $[t(1,49)=0.47, p>$.6] or accuracy $[t(1,49)=0.29, p>.7]$.

Orienting. The first contrast between the MBSR group and the control group revealed no significant difference across groups in either RT difference $[t(1,32)=0.39 . p>$ .7] or accuracy difference $[t(1,32)=0.18, p>$.8] scores. In the second contrast, these groups were collapsed and their performance was compared with the performance of the retreat group. No significant differences across these groups were present for RT $[t(1,49)=1.1, p>.2]$ or accuracy $[t(1,49)=1.02, p>.3]$ scores.

Conflict monitoring. The first contrast between the MBSR group and the control group revealed no significant difference across groups in either RT difference $[t(1,32)=0.26, p>.8]$ or accuracy difference $[t(1,34)=$ $0.07, p>.9]$ scores. In the second contrast, these groups were collapsed and their performance was compared with the performance of the retreat group. This contrast revealed a significant difference across groups for RT $[t(1,49)=2.22, p<.03]$ and accuracy $[t(1,49)=5.62$, $p<.001]$. As is depicted in Figure 2, the magnitude of conflict monitoring was reduced in the retreat relative to the control + MBSR participants. Thus, there was a reduction in flanker interference for the retreat participants relative to the control + MBSR participants, which is consistent with previous reports of the influence of meditation on Stroop interference (see Wenk-Sormaz, 2005, for a review).

\section{Time 2: The Influence of Mindfulness Training}

Does MT alter dorsal system functioning? To investigate the hypothesis that MT may improve the ability to endogenously orient attention during input selection, response selection, or both stages of analysis, orienting and conflict monitoring difference scores at Time 2 were examined. An ANOVA for the RT difference scores of the orienting component revealed a significant factor of group $[F(2,48)=10.13, p<.001]$. A series of contrasts revealed that the MBSR group differed from the control group (Bonferroni-adjusted $p=.046$ ) and the retreat group (Bonferroni-adjusted $p<.001$ ). However, the retreat and control groups did not differ from each other (Bonferroni-adjusted $p=.161$ ). There was no significant effect of group on the accuracy difference scores $(p>.39)$.

Since the RT difference scores from the retreat and control groups did not significantly differ from each other, their scores were combined for illustrative purposes in Figure 3. Figure $3 \mathrm{~A}$ depicts the orienting difference scores 


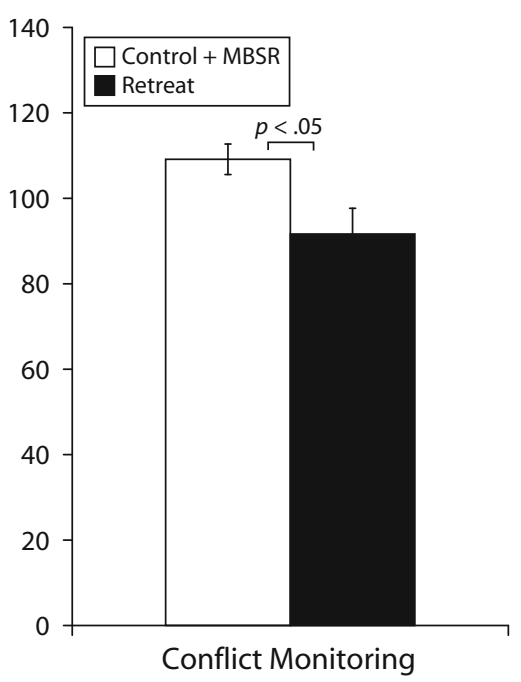

Figure 2. Difference scores for conflict monitoring components at Time 1 for the retreat group (filled bar) versus the control + MBSR group (unfilled bar). The only component on which these two groups differed significantly was the conflict monitoring component. The magnitude of the conflict monitoring effect was significantly reduced for the retreat group relative to the control + MBSR group.

for the MBSR group and the control + retreat group. The magnitude of orienting is greater for the MBSR group. However, this pattern cannot reveal which condition(s) (center cue, spatial cue, or both) was altered with training. Figure 3B depicts the RTs on center-cue and spatialcue trials for MBSR versus control + younger retreat groups. RTs for the younger retreat subgroup were plot- ted instead of RTs for the entire retreat group because of age-related slowing in RTs in the older versus the younger retreat participants (see the section on Influence of Age, above). A paired post hoc comparison across MBSR versus control + younger retreat groups revealed that RTs were nearly significantly shorter in the MBSR group than in the control + younger retreat group in the spatial-cue condition $(p<.08)$ but not in the center-cue condition $(p>.8)$. When the entire retreat group was included, the results for the spatial-cue condition were in the same direction and significant $(p<.01)$, but those for the centercue condition were not $(p>.34)$.

An ANOVA for the conflict monitoring component RT and accuracy difference scores revealed no significant main effects and no significant effects on any of the paired contrasts $(p>.36)$.

Finally, to investigate the homogeneity of variance of the three groups in their orienting and conflict scores, we performed Levene's test for homogeneity of variance. There were no significant differences across groups for either orienting or conflict monitoring scores for either RT or accuracy $(p>.21)$.

Does MT alter ventral system functioning? To investigate the hypothesis that retreat participation may allow for the emergence of improved exogenous stimulus detection, alerting scores at Time 2 were examined in a series of planned contrasts. The first contrast between the MBSR and control groups revealed no significant difference across groups in either RT or accuracy difference scores $(p>.19)$. The second contrast, collapsed across these groups, was performed to compare the performance of the participants in these groups to that of the participants in the retreat group. There was a significant differ-
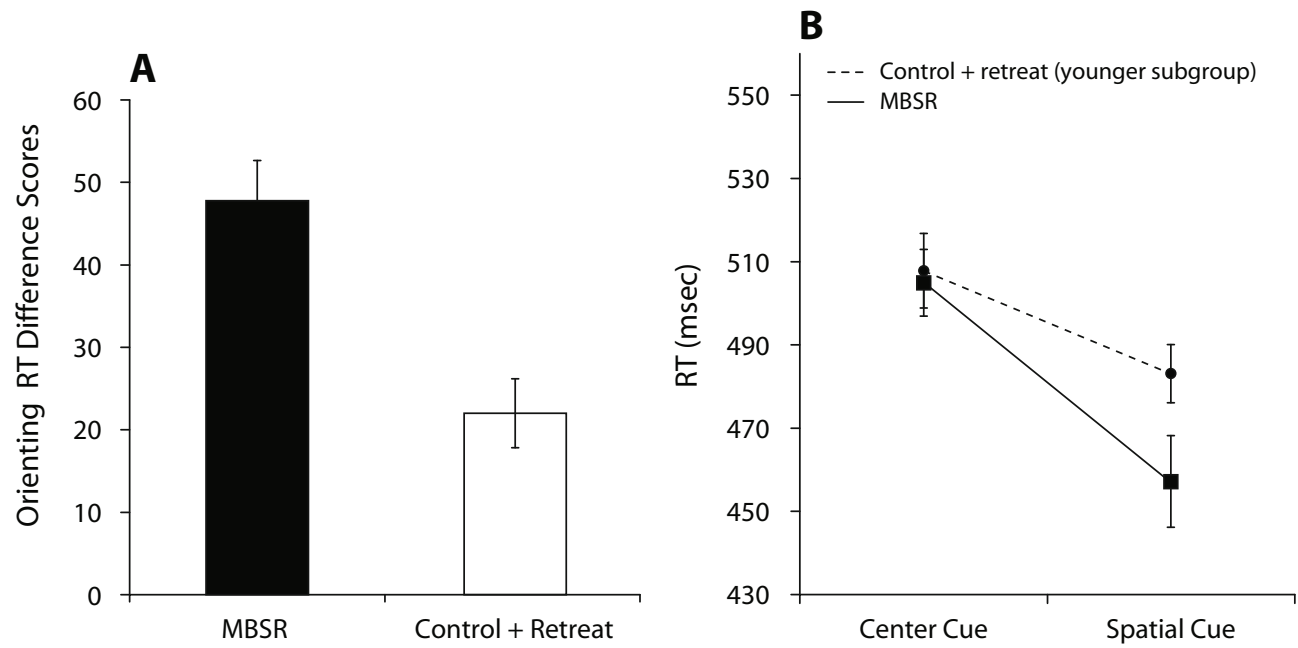

Figure 3. (A) Difference scores for the orienting component computed by subtracting response times (RTs, in milliseconds) on spatial-cue trials from RTs on center-cue trials, for the MBSR group (filled bar) versus the control + retreat group (unfilled bar). The orienting difference score is significantly greater for the MBSR group. (B) Time 2 RTs (in milliseconds) for the MBSR group (filled squares) and the control + retreat (younger) group (filled circles) in the center-cue and spatial-cue conditions of the orienting component. Although RTs do not differ between these two groups for the center-cue trials, they are nearly significantly shorter for the MBSR group than for the control + retreat group on the spatial-cue trials $(p<.08)$. 

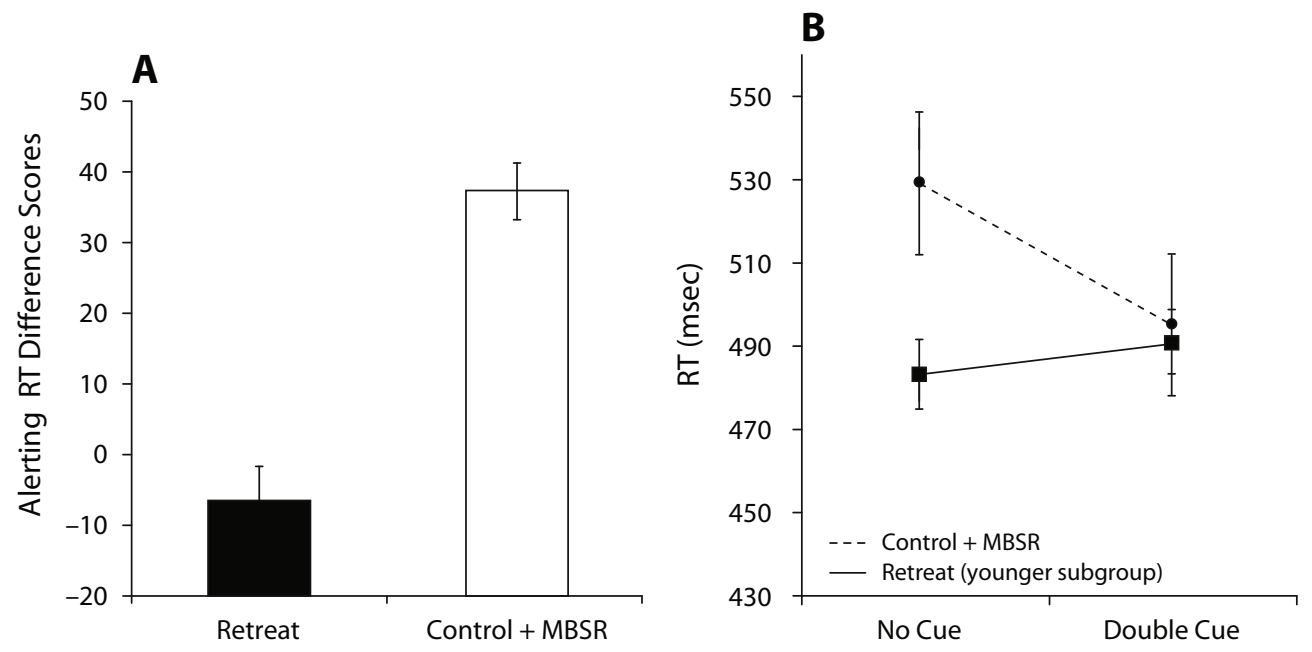

Figure 4. (A) Difference scores for the alerting component computed by subtracting response times (RTs, in milliseconds) on double-cue trials from RTs on no-cue trials, for the retreat group (filled bar) versus the control + MBSR group (unfilled bar). The alerting difference score is significantly smaller for the retreat group. (B) Time 2 RTs (in milliseconds) for the younger retreat group (filled squares) and the control + MBSR group (filled circles) in the no-cue and double-cue conditions of the alerting component. Although RTs do not differ between these two groups for the double-cue trials, they are significantly shorter for the retreat group on the no-cue trials.

ence in RT scores $[t(1,49)=3.55, p<.001]$ but not in accuracy scores $(p>.7)$. As is depicted in Figure 4A, the magnitude of the retreat scores was reduced in the retreat group in comparison with the control + MBSR group. Figure 4B depicts the RTs on no-cue and double-cue trials for both groups. A paired comparison across younger retreat versus control + MBSR groups revealed that nocue RTs were significantly shorter in the younger retreat group than in the control + MBSR group $[t(1,40)=2.17$, $p<.03$ ], but double-cue RTs were not $(p>.6)$.

\section{Time 1 Versus Time 2: Influence of Task Repetition on Performance}

An ANOVA examining two factors-Time (1 vs. 2) and group (retreat vs. MBSR vs. control) — on overall RT and accuracy measures (collapsed across all conditions) revealed no main effect of group $(p>.3)$. However, there was a significant main effect of time $[F(1,16)=13.6, p<$ $.001]$ in that RTs were faster at Time 1 than at Time 2 . No significant interaction effects were observed $(p>.17)$. In addition, no significant effects were observed for accuracy scores $(p>.2)$. This analysis confirms that, since there was an overall speeding of RTs over time, comparisons across groups at each time point (e.g., MBSR vs. control at Time 2), as conducted above, may be most appropriate for revealing MT-related effects.

\section{Influence of Age in the Retreat Group}

A paired contrast between younger $(n=8$, mean age $=23$ years, $S D=3)$ and older $(n=9$, mean age $=$ 45 years, $S D=8$ ) participants revealed that RTs were nearly significantly faster for the younger than for the older participants at Time $1[t(1,15)=2.0, p<.06]$ and were significantly faster for the former group at Time 2 $[t(1,15)=2.6, p<.01]$. This is consistent with several previous studies, which also showed increases in RTs with increasing age (see Colcombe et al., 2004). No significant differences in accuracy were observed across age groups at either time point $(p>.2)$. RT and accuracy difference scores for younger versus older retreat subgroups did not significantly differ at Time $1(p>.1)$ or Time $2(p>.4)$ for any of the subcomponents (alerting, orienting, and conflict monitoring). It is important to note that the mean age of the younger group did not differ significantly from the mean age of the MBSR or the control group $(p>.35)$. For this reason, the RTs of only the younger retreat participants were plotted in Figures 3B and 4B when explicit RT comparisons were made.

\section{Influence of MT As a Function of Meditation Experience in the Retreat Group}

Since only the alerting component significantly changed at Time 2 for the retreat group, we examined whether or not the months of meditation experience of the retreat participants correlated with the magnitude of the alerting score at Time 2 . The correlation value $(R=-.52)$ was significant $(t=2.4, p<.03)$. Thus, there was a significant reduction in alerting score with increased meditation experience in the retreat group.

\section{DISCUSSION}

In this study, we examined the influence of MT on the functioning of specific attentional subsystems. Participants receiving MT in the form of MBSR or retreat experience performed the ANT before and after training. Their performance was contrasted with the performance of control participants who were also tested at two time 
points. We investigated two main hypotheses: (1) We hypothesized that prior experience with - and training inconcentrative meditation techniques would correspond to greater efficiency in the functioning of the dorsal attention system, which subserves voluntary top-down attentional selection. Positive support for this hypothesis was observed in two respects. First, the retreat participants, who were experienced with concentrative meditation at Time 1, demonstrated better conflict monitoring (reduced flanker interference) in RT and accuracy performance than did the meditation-naive participants. Second, after MT at Time 2, the MBSR participants improved in their orienting performance relative to the control participants. Since both conflict monitoring and orienting are forms of voluntary attentional selection, our results suggest that concentrative meditation may indeed alter functioning of the dorsal attention system to improve voluntary responseand input-level selection processes. (2) We hypothesized that prior experience with concentrative meditation may allow for the emergence of receptive attention after MT. Receptive attention corresponds to ventral attention system functions of exogenous stimulus detection as well as attentional readiness and alerting. We found that after MT, not only did the retreat participants differ in their alerting performance from the control + MBSR participants, but the magnitude of their alerting scores at Time 2 was correlated with their prior meditation experience. Greater experience corresponded to reduced alerting scores, indicating that attention was in a more readied state when no warning about target onset was provided. Thus, our results suggest that MT improves performance on specific conditions of the ANT. Although provocative, many questions about these results still remain and many future studies are needed.

If concentrative meditation improves voluntary attentional selection, why weren't comparable attentional effects observed at Time 1 (in experienced meditators) and Time 2 (in the MBSR and retreat groups)? There are several possibilities to consider. Improvement in conflict monitoring performance was observed in the retreat group relative to the meditation-naive groups at Time 1 . Nevertheless, no differences in conflict monitoring were observed among the MBSR, retreat, and control participants at Time 2. This result was surprising given that several previous reports have suggested improvements in conflict monitoring with meditation (see Wenk-Sormaz, 2005, for a review). We performed a post hoc analysis on the control participants' conflict monitoring scores over time. It revealed that there was an improvement in conflict monitoring at Time 2 relative to Time 1 in the absence of any attention training. A possible explanation for this improvement is that the ANT may be highly susceptible to task exposure effects for the conflict monitoring component (Michael Posner, personal communication, November 2005). Thus, the lack of a groupwise difference in conflict monitoring performance at Time 2 may have occurred because all groups improved. Even if the two MT groups improved above and beyond levels resulting from simple task exposure benefits, such improvement may have been masked by floor effects in RT performance. The ANT is an elegant and powerful tool that has been useful in demonstrating attentional training effects in children (see Rueda, Rothbart, McCandliss, Saccomanno, \& Posner, 2005), yet it may lack sensitivity to index some MT-related changes in adults. As such, in future studies tasks that allow for a precise titration of task exposure versus MT effects should be used.

Another possibility is that there may be subtle differences in concentrative attention instructions conveyed during various meditation contexts. The MBSR participants received highly structured instruction (see KabatZinn, 1994) whereas the retreat group relied on informal and self-paced instructions to guide concentrative meditation prior to entry into the retreat. During the retreat, this group received formal but minimal instructions in concentrative attention, and most of the retreat was held in silence (see Trungpa, 1975). Whereas the present study relied on extant MT protocols that are widely available, future studies could explicitly manipulate the "dose" and emphasis of concentrative instruction in MT to examine attentional effects.

Since concentrative attention was part of the meditation instruction for both MT groups, it was perplexing that orienting scores were improved in the MBSR relative to the retreat group at Time 2. One possibility, as discussed above, concerns subtle differences in instructions. A second possibility is that the retreat participants may not have adhered to the instructions in the same fashion throughout the retreat. For example, during the early portion of the retreat, participants may have engaged concentrative attention as instructed. At some later point during the retreat, they may have discovered that they were able to hold attention in a receptive and open fashion without mind wandering (see Smallwood \& Schooler, 2006). Once they successfully noticed the emergence of receptive attention, they may have chosen to engage in receptive instead of concentrative meditation for the remainder of the retreat.

Consistent with this conjecture, we found that the retreat participants demonstrated a reduction in their alerting scores at Time 2 relative to the control + MBSR participants. Group differences were driven by RTs in the no-cue condition, which were faster in the younger retreat subgroup than in the comparably aged control + MBSR group. No significant differences in the double-cue condition were observed across groups at Time 2. It is important to note that the reduction in alerting scores for the retreat group does not indicate that this group was less alert. Instead, these results suggest that after MT, at Time 2, the participants in the retreat group were able to more efficiently and easily detect targets when no information was provided about where or when they would appear. One interpretation of these results is that their attention was in a more readied state. Attentional readiness in the absence of a selective focus is akin to receptive attention as described in meditation texts (see Lutz et al., in press). Thus, the present results provide positive support that MT may have resulted in the emergence of receptive attention in the meditation-experienced retreat group.

In line with the present findings, many meditation texts describe a practice-related trajectory of the effects of meditation on attention (see Lutz et al., in press), with an 
initial development of concentrative attention in the novice meditator and cultivation of receptive attention in the experienced meditator. Many future studies are necessary to determine the behavioral and neural mechanisms by which distinct attentional changes may come about with MT. We discuss some possibilities below.

How might MT entrain the dorsal attention system to improve attentional functioning? Training of concentrative attention includes instructions to select a specific attentional focus, such as the breath, and maintain attention on that focus. When attention moves to nonbreath stimuli, it is to be disengaged and gently returned to the breath. The practice of repeatedly engaging, moving, and disengaging instantiates the orienting or "shift" operation of attention (Posner \& Badgaiyan, 1998; Posner \& Gilbert, 1999). Cognitive neuroscience investigations of attentional orienting use spatial cuing paradigms to index the neural bases of attention. Using these paradigms, numerous studies have now identified neural systems supporting this attentional subsystem with prominent activations observed within dorsal prefrontal and posterior parietal cortices (Corbetta, Kincade, \& Shulman, 2002). Just as the breath serves as the concentrative focus during MT, the spatial cue directs concentrative focus toward the location indicated by the cue during performance of the spatial cuing paradigms. Participants are instructed to engage attention at the cue location, move attention to that location, and disengage attention if the target does not appear at the cued position. The ANT, which was used in the present study, has only valid cues and never explicitly requires disengagement during invalid cue trials. Nonetheless, performance improvements in the orienting component, and specifically the spatial-cue trials, for the MBSR group could be due to improved efficiency of the "engage" component of the orienting subsystem. This improvement may result from repeatedly engaging, moving, and disengaging attention during MT. Importantly, this speculation relies on an assumption of generalizability across task contexts such that improvements in orienting via MT transfers to orienting benefits during visuospatial computer tasks.

It is important to note that concentrative meditation practices were performed for only a small fraction of the MBSR participants' day ( $30 \mathrm{~min})$. How might such a limited-duration activity impact functioning of the dorsal attention system? Recent fMRI studies have suggested that this system may be identifiable not only during fMRI responses to external attentional demands, but also in the internal dynamics of spontaneous brain activity when the brain is at rest (Fox, Corbetta, Snyder, Vincent, \& Raichle, 2006). Correlations in spontaneous fluctuations of fMRI signal in all regions comprising the dorsal attention network in the absence of task, stimuli, or explicit attentional demands were highly correlated with each other. In addition, a similar coherence in spontaneous correlations was observed among subregions comprising the ventral attention system. One speculation is that the practice of meditation for even short durations could be altering the default functioning of these attentional systems by modi- fying their internal dynamics. Yet, no studies to date have been conducted to investigate whether or not the degree of coherence of dorsal and ventral attention systems corresponds to the functioning of these systems. Thus, many questions remain regarding the nature of default coherence in these systems as a result of behavioral training, pharmacologic treatment, development, aging, or disease states.

How might noncognitive factors contribute to the pattern of results observed in the present study? Most individuals in MBSR courses participate in them to reduce stress in their lives. Although stress reduction is a proven benefit of MT, the mechanism through which MT facilitates stress reduction is still under active debate (Baer, 2003). Some have suggested that a key component of MT is the ability to elicit the relaxation response that counteracts the stress response (Benson, 1975). Others argue that MT's utility is in cognitive training of attention to allow for re-interpretation of stressors (Teasdale, Segal, \& Williams, 1995). Nevertheless, it is possible that MT does not directly improve attention but that physiological effects, such as a reduction in autonomic nervous system "fight-or-flight" functions mediate the attentional effects of MT described here. Careful studies in which the contribution of stress reduction and attention training is parsed with mediation analyses, for example, have not yet been reported.

Finally, the present results must be interpreted cautiously and considered preliminary since the experiment reported here is one of the first conducted to evaluate the relationship between MT and attentional subsystems. In addition, although our results were reliable and significant, our training groups included relatively small numbers of participants. Nonetheless, these results are consistent with the view that MT may entrain attention in specific and enduring ways. We view the present results as being in line with many prior demonstrations of training-related improvements in the functioning of attention. In neurologically normal participants, for example, video game playing (Green \& Bavelier, 2003) and exercise (Colcombe et al., 2004) result in improvements in specific attentional subsystems. There is also growing support that neurologically impaired participants can improve attentional functioning with training (Robertson, Tegnér, Tham, Lo, \& Nimmo-Smith, 1995; Sohlberg \& Mateer, 1987). Many future studies are needed to develop a better understanding of the relationship between MT and attention. We anticipate that future inquiry will build upon the present study by including an active-training control group, neural measurements to accompany behavioral measures, and more elaborated characterization and control over training protocols. Nonetheless, we believe that the present findings are a provocative demonstration of MT as an attention-training protocol. Attention is central to many higher order cognitive operations, susceptible to dysfunction during normal aging, and impaired in many disease states (e.g., attention deficit disorder). As such, the ability to improve attention with training methods has the potential to be beneficial in many domains. 


\section{AUTHOR NOTE}

This research was supported by NIH Grant NCCAM 1 R21 AT002761 and funding from the Penn Program for Stress Management. We thank Acharya Bill McKeever and the staff of Shambhala Mountain Center for their cooperation during the execution of this project. In addition, we thank Dave Meyer, Clifford Saron, Ron Mangun, Michael Posner, Patricia Reuter-Lorenz, Ruth Baer, Saul Sternberg, and two anonymous reviewers for helpful comments on earlier drafts, and Rebecca Klein, Audrey Lustig, Kartik Sreenivasen, Ling Wong, Marieke Van Vugt, Adam Safron, Gopal Shah, Zev Rosen, and Erik Malmgren for engaging discussions and assistance with data coding and analysis. Correspondence concerning this article should be addressed to A. P. Jha, Center for Cognitive Neuroscience, Department of Psychology, University of Pennsylvania, 3401 Walnut St., Suite 302C, Philadelphia, PA 19104 (e-mail: apjha@psych.upenn.edu).

\section{REFERENCES}

Astin, J. A. (1997). Stress reduction through mindfulness meditation: Effects on psychological symptomatology, sense of control, and spiritual experiences. Psychotherapy \& Psychosomatics, 66, 97-106.

BAER, R. A. (2003). Mindfulness training as a clinical intervention: A conceptual and empirical review. Clinical Psychology: Science \& Practice, 10, 125-143.

BENSON, H. (1975). The relaxation response. New York: Morrow.

Bishop, S. R., Lau, M., Shapiro, S., Carlson, L., Anderson, N. D., CARMody, J., ET AL. (2004). Mindfulness: A proposed operational definition. Clinical Psychology: Science \& Practice, 11, 230-241.

Broadbent, D. E. (1970). Stimulus set and response set: Two kinds of selective attention. In D. I. Mostofsky (Ed.), Attention: Contemporary theory and analysis (pp. 51-60). New York: Appleton-Century-Crofts.

Brown, D. P. (1977). A model for the levels of concentrative meditation. International Journal of Clinical \& Experimental Hypnosis, 25, 236-273.

BroWn, D. [P.], Forte, M., \& Dysart, M. (1984). Visual sensitivity and mindfulness meditation. Perceptual \& Motor Skills, 58, 775-784.

Colcombe, S., Kramer, A., Erickson, K., Scalf, P., McAuley, E., Cohen, N., ET AL. (2004). Cardiovascular fitness, cortical plasticity, and aging. Proceedings of the National Academy of Sciences, 101, 3316-3321.

Corbetta, M., Kincade, J. M., \& Shulman, G. L. (2002). Neural systems for visual orienting and their relationships to spatial working memory. Journal of Cognitive Neuroscience, 14, 508-523.

Corbetta, M., \& Shulman, G. L. (2002). Control of goal-directed and stimulus-driven attention in the brain. Nature Reviews Neuroscience, 3, 201-215.

DELMONTE, M. M. (1987). Meditation: Contemporary theoretical approaches. In M. A. West (Ed.), The psychology of meditation (pp. 3958). Oxford: Oxford University Press, Clarendon Press.

Fan, J., Fossella, J., Sommer, T., Wu, Y., \& Posner, M. I. (2003). Mapping the genetic variation of executive attention onto brain activity. Proceedings of the National Academy of Sciences, 100, 7406-7411.

Fan, J., McCandliss, B. D., Fossella, J., Flombaum, J. I., \& Posner, M. I. (2005). The activation of attentional networks. NeuroImage, 26, 471-479.

Fan, J., McCandliss, B. D., Sommer, T., Raz, A., \& Posner, M. I. (2002). Testing the efficiency and independence of attentional networks. Journal of Cognitive Neuroscience, 14, 340-347.

Forte, M., Brown, D. P., \& DysarT, M. (1987-1988). Differences in experience among mindfulness meditators. Imagination, Cognition, \& Personality, 7, 47-60.

Fossella, J., Posner, M., Fan, J., Swanson, J., \& Pfaff, D. (2002). Attentional phenotypes for the analysis of higher mental function. Scientific World, 2, 217-223.

Fox, M. D., Corbetta, M., Snyder, A. Z., Vincent, J. L., \& Raichle, M. E. (2006). Spontaneous neuronal activity distinguishes human dorsal and ventral attention systems. Proceedings of the National Academy of Sciences, 103, 10046-10051.

GREEN, C. S., \& BAVELIER, D. (2003). Action video game modifies visual selective attention. Nature, 423, 534-537.
Grossman, P., Niemann, L., Schmidt, S., \& Walach, H. (2004). Mindfulness-based stress reduction and health benefits: A metaanalysis. Journal of Psychosomatic Research, 57, 35-43.

KaBAT-ZinN, J. (1990). Full catastrophe living: Using the wisdom of your body and mind to face stress, pain, and illness (15th anniversary ed.). New York: Bantam Doubleday Dell.

Kabat-ZINN, J. (1994). Mindfulness meditation for everyday life. New York: Hyperion.

Kabat-ZinN, J. (2003). Mindfulness-based interventions in context: Past, present, and future. Clinical Psychology: Science \& Practice, 10, 144-156.

KABAT-ZINN, J., Lipworth, L., \& BuRney, R. (1985). The clinical use of mindfulness meditation for the self-regulation of chronic pain. Journal of Behavioral Medicine, 8, 163-190.

Kabat-Zinn, J., Massion, A. O., Kristeller, J., Peterson, L. G., Fletcher, K. E., Pbert, L., et Al. (1992). Effectiveness of a meditation-based stress reduction program in the treatment of anxiety disorders. American Journal of Psychiatry, 149, 936-943.

Kapleau, P. (1965). The three pillars of Zen: Teaching, practice, and enlightenment. Boston: Beacon Press.

Kristeller, J. L., \& Hallett, C. B. (1999). An exploratory study of a meditation-based intervention for binge eating disorder. Journal of Health Psychology, 4, 357-363.

LutZ, A., Dunne, J. P., \& DAVIDSon, R. J. (in press). Meditation and the neuroscience of consciousness: An introduction. In P. D. Zelazo, M. Moscovitch, \& E. Thompson (Eds.), Cambridge handbook of consciousness. New York: Cambridge University Press.

Marlatt, G. A., \& Kristeller, J. L. (1999). Mindfulness and meditation. In W. R. Miller (Ed.), Integrating spirituality into treatment: Resources for practitioners (pp. 67-84). Washington, DC: American Psychological Association.

Page, R. C., McAuliffe, E., Weiss, J. F., Ugyan, J., Wright, L. S., \& Maclachlan, M. (1997). Self-awareness of participants in a longterm Tibetan Buddhist retreat. Journal of Transpersonal Psychology, 29, 85-98.

PfeIfFer, W. M. (1966). Konzentrative Selbstentspannung durch Übungen, die sich aus der buddhistischen Atemmeditation und aus der Atemtherapie herleiten [Concentrative self-relaxation by exercises derived from Buddhistic respiration-meditation and from respiration therapy]. Zeitschrift für Psychotherapie \& Medizinische Psychologie, 16, 172-181.

Posner, M. I., \& Badgaiyan, R. D. (1998). Attention and neural networks. In R. W. Parks, D. S. Levine, \& D. L. Long (Eds.), Fundamentals of neural network modeling: Neuropsychology and cognitive neuroscience (pp. 61-76). Cambridge, MA: MIT Press.

Posner, M. I., \& Gilbert, C. D. (1999). Attention and primary visual cortex. Proceedings of the National Academy of Sciences, 96, 2585-2587.

Posner, M. I., \& Petersen, S. E. (1990). The attention system of the human brain. Annual Review of Neuroscience, 13, 25-42.

Reuter-Lorenz, P. A., \& STANCZAK, L. (2000). Differential effects of aging on the functions of the corpus callosum. Developmental Neuropsychology, 18, 113-137.

Robertson, I. H., Tegnér, R., Tham, K., Lo, A., \& Nimmo-Smith, I. (1995). Sustained attention training for unilateral neglect: Theoretical and rehabilitation implications. Journal of Clinical \& Experimental Neuropsychology, 17, 416-430.

RuEdA, M. R., Rothbart, M. K., McCandliss, B. D., Saccomanno, L., \& Posner, M. I. (2005). Training, maturation, and genetic influences on the development of executive attention. Proceedings of the $\mathrm{Na}$ tional Academy of Sciences, 102, 14931-14936.

SEMPLE, R. J. (1999). Enhancing the quality of attention: A comparative assessment of concentrative meditation and progressive relaxation. Unpublished Master's thesis, University of Auckland, New Zealand.

Smallwood, J., \& Schooler, J. W. (2006). The restless mind. Psychological Bulletin, 132, 946-958.

SOHLBERG, M. M., \& MATEER, C. A. (1987). Effectiveness of an attentiontraining program. Journal of Clinical \& Experimental Neuropsychology, 9, 117-130

Speca, M., Carlson, L. E., Goodey, E., \& Angen, M. (2000). A randomized, wait-list controlled clinical trial: The effect of a mindfulness 
meditation-based stress reduction program on mood and symptoms of stress in cancer outpatients. Psychosomatic Medicine, 62, 613-622.

Speeth, K. R. (1982). On psychotherapeutic attention. Journal of Transpersonal Psychology, 14, 141-160.

Teasdale, J. D., Segal, Z. [V.], \& Williams, J. M. G. (1995). How does cognitive therapy prevent depressive relapse and why should attentional control (mindfulness) training help? Behaviour Research \& Therapy, 33, 25-39.

Teasdale, J. D., Segal, Z. V., Williams, J. M. G., Ridgeway, V. A., Soulsby, J. M., \& LaU, M. A. (2000). Prevention of relapse/recurrence in major depression by mindfulness-based cognitive therapy. Journal of Consulting \& Clinical Psychology, 68, 615-623.

ToRI, C. D. (1999). Change on psychological scales following Buddhist and Roman Catholic retreats. Psychological Reports, 84, 125-126.
Trungra, C. (1975). The myth of freedom and the way of meditation. Boston: Shambhala.

Valentine, E. R., \& Sweet, P. L. G. (1999). Meditation and attention: A comparison of the effects of concentrative and mindfulness meditation on sustained attention. Mental Health, Religion, \& Culture, 2, 59-70.

Wallace, B. A. (1999). The Buddhist tradition of Samatha: Methods for refining and examining consciousness. Journal of Consciousness Studies, 6, 175-187.

WENK-SORMAZ, H. (2005). Meditation can reduce habitual responding. Advances in Mind-Body Medicine, 21, 33-49.

(Manuscript received October 5, 2005;

revision accepted for publication December 4, 2006.) 